\title{
Your country is your routine: the evacuation, quarantine, and management of behavioral problems of a child with autism during COVID-19 pandemic
}

\author{
Bahadir Turan $^{1 \oplus}$, Nergiz Kocarslan $^{2 \oplus}$, Murat Gulsen $^{1 \oplus}$, Onur Burak Dursun ${ }^{1 \odot}$ \\ ${ }^{1}$ Turkish Ministry of Health, General Directorate of Health Services, Autism, Department of Mental Special Needs and Rare Diseases, \\ Ankara - Turkey \\ ${ }^{2}$ Private Practice, Istanbul - Turkey
}

\begin{abstract}
As the coronavirus disease 2019 (COVID-19) outbreak began to spread around the globe, countries reacted to the threat with a series of measures implemented in varying degrees. The Special Children Support System of Turkey was designed to provide multidisciplinary support for the families of children with developmental special needs who experience difficulty during COVID-19 pandemic and related home confinement. The system combines telehealth applications with services from local psychosocial intervention teams. This report describes the case of an 11-year old child with autism spectrum disorder who returned to Turkey from the United Kingdom and was housed in a dormitory for a mandatory 14-day quarantine period. The telehealth-based system developed by our institution specifically to support children with special needs during the COVID-19 pandemic was a tool the family used to help manage the circumstances. Changes in the environment and routine can be very difficult for those with autism. It is important that appropriate intervention mechanisms are available and used early. The intensive application of both behavioral and medical treatment with a multidisciplinary team resulted in a significant change in the patient's symptoms, Access to and use of a rapid intervention system can have a decisive impact.
\end{abstract}

Keywords: Autism spectrum disorder, COVID-19, integrative psychiatry

\section{INTRODUCTION}

The coronavirus disease 2019 (COVID-19) pandemic has led both countries and individuals to adopt various precautions to control the spread of the disease. One of the earliest measures used by several nations, including Turkey, was the termination of all scheduled international flights (1). This decision led to some people becoming stranded in a foreign location amid the pandemic. Some governments made arrangements to evacuate and repatriate their citizens. Turkey evacuated some 29.000 nationals from abroad. As part of the process, after landing in Turkey, mandatory health checks were performed and a 14-day quarantine in government housing was implemented (1).

A telehealth-based system developed by our institution specifically to support children with special needs during the COVID 19 pandemic was instrumental

How to cite this article: Turan B, Kocarslan N, Gulsen M, Dursun OB. Your country is your routine: the evacuation, quarantine, and management of behavioral problems of a child with autism during COVID-19 pandemic. Dusunen Adam The Journal of Psychiatry and Neurological Sciences 2020;33:310-312.

Correspondence: Onur Burak Dursun, Turkish Ministry of Health, General Directorate of Health Services, Autism, Department of Mental Special Needs and Rare Diseases, 06800, Ankara - Turkey

E-mail: onurburak007@yahoo.com

Received: June 29, 2020; Revised: August 04, 2020; Accepted: August 22, 2020 
in the care of an 11-year old child with autism spectrum disorder who returned from the United Kingdom to Turkey during the pandemic and was held in a dormitory for a 14-day quarantine period.

\section{CASE}

An 11-year-old boy had been diagnosed with autism spectrum disorder at 3 years of age. The patient's family lived in Turkey, however, he was in the United Kingdom with his family when the COVID-19 pandemic spread to Europe and they were unable to return home before international flights between the countries were discontinued as a control measure. The family applied to the Turkish Ministry of Foreign Affairs for evacuation and their application was accepted. The boy became irritable on the flight, and began to scream and hit himself.

Upon arrival, the family underwent a required medical check-up and was transferred to a dormitory for a 14-day quarantine period. In the dorm, the boy had a tantrum, and the family contacted the government for behavioral support. They were directed to a new service called the Special Children Support System (SCSS), which was developed by our institution to assist with managing the behavioral problems of children with special needs during the COVID-19 pandemic.

The SCSS mobile application makes it possible for children with special needs and their families to participate in remote video calls to address added misbehavior and difficulties resulting from the constrictions and changes experienced due to the pandemic. Once the application is downloaded and open, callers are first greeted by operators who can refer families to trained counselors in applied behavior analysis or child and adolescent psychiatrists for psychiatric help and psychotherapy.

In the first video call with the case patient's family, a detailed history and information about current symptoms were collected. The patient was a full-term infant, delivered with no complications. His mother reported that during infancy and as a toddler, he was healthy and his motor development was within the normal limits for the milestones of sitting, standing, and walking. At age 3 he was described as a low muscle tone with awkward motor skills and inconsistent imitation skills. His communication development was delayed; he began using vocalizations at 3 months of age but had developed no words by 3 years. He had been in a special education program at a rehabilitation center for over 8 years and was taking risperidone $3 \mathrm{mg} /$ day for irritability and melatonin $1.5 \mathrm{mg} /$ day for sleep difficulties. Observation of the boy was attempted during the video call, but at the time, he was irritable, he did not want to participate, and his stereotypical movements were progressing.

In the second visit, his counselor continued to work with the family. They first addressed establishing routines. Then, they worked on behavioral analysis and techniques to reinforce positive behaviors and ignore negative behaviors. They also discussed activities the boy could do in the dorm. A referral to a SCSS child and adolescent psychiatrist was arranged to discuss his medication.

In follow-up visits, the psychiatrist adjusted the risperidone treatment to $2 \mathrm{mg}$ in the morning and $1 \mathrm{mg}$ in the evening. Melatonin was increased to $3 \mathrm{mg} /$ day taken in the evening. Family sessions with the counselor were continued.

Acute management yielded a significant improvement in the first week. Behavioral problems and irritability decreased and he developed a regular sleep pattern. At the end of the fourth day, the family reported they were almost as comfortable as they were at home.

Written, informed consent for publication was obtained from the parents of the patient.

\section{DISCUSSION}

Isolation and quarantine conditions, separation from loved ones, loss of freedom, and uncertainty about the course of the pandemic can have dramatic effects on people (2). Problems like irritability, related behavioral issues, and communication difficulties may be observed $(2,3)$. Acute stress responses appear in the first days, and may be followed by adjustment disorder with a depressed temperament and behavior problems (3). Restriction to an indoor environment can also lead to exacerbation or aggravation of the symptoms of an individual with a mental disorder (3). Due to the nature of autism, changes to the environment and routine can provoke negative effects; therefore, interventions must be ready and implemented early (4). The intensive application of both behavioral and medical treatment with a multidisciplinary team led to a significant change in our patient's symptoms very quickly. Access to a system that is equipped to provide rapid intervention can have a dramatic and decisive impact.

The evidence suggests that best practice for managing maladaptive behavior in children with special needs is a combination of behavioral interventions and psychiatric support (5). However, implementation of this best practice while under pandemic conditions was unclear. In this context, we used a 3-stage, integrative telehealth model. Applied behavioral analysis is generally used as 
the most effective tool for first-line maladaptive behavior management (6). In the SCSS model, a child psychiatrist consultation and medication were second-line considerations for cases with more complicated problems. Local units were also established as a thirdline measure for cases that could not be sufficiently addressed via telehealth. A multilayered system that has a strong technical infrastructure, enables the early implementation of multidisciplinary intervention to support individuals with special needs, especially in acute and challenging conditions. A general limitation of telehealth services, especially models involving caregiver facilitation, is that the quality and intensity of services may be limited based on caregiver availability. Although this potential limitation is a significant concern, if face-to-face services are not possible, as in this case, due to the COVID-19 outbreak, properly substantiated telehealth services can provide substantial benefits to the patient (7-9).

\begin{tabular}{|c|c|c|}
\hline \multicolumn{2}{|c|}{ Contribution Categories } & \multirow{2}{*}{$\begin{array}{l}\text { Author Initials } \\
\text { O.B.D., B.T. }\end{array}$} \\
\hline \multirow{4}{*}{ Category 1} & Concept/Design & \\
\hline & Literature review & B.T., M.G. \\
\hline & Data analysis/Interpretation & O.B.D., B.T. \\
\hline & Case follow-up (if applicable) & B.T., N.K. \\
\hline \multirow{2}{*}{ Category 2} & Drafting manuscript & B.T. \\
\hline & Critical revision of manuscript & M.G., B.T. \\
\hline Category 3 & Final approval and accountability & O.B.D., B.T. \\
\hline \multirow{2}{*}{ Other } & Technical or material support & M.G., N.K. \\
\hline & Supervision & O.B.D. \\
\hline
\end{tabular}

Informed Consent: Written informed consent of the patient has been obtained from the parents.

Peer-review: Externally peer-reviewed.

Conflict of Interest: None declared.

Financial Disclosure: None declared.

\section{REFERENCES}

1. Republic of Turkey Ministry of Health. COVID-19. https://www. saglik.gov.tr/. Accessed August 17, 2020.

2. Huremović D. Psychiatry of pandemics: a mental health response to infection outbreak. Springer, 2019.

3. Brooks SK, Webster RK, Smith LE, Woodland L, Wessely S, Greenberg N, et al. The psychological impact of quarantine and how to reduce it: rapid review of the evidence. The Lancet 2020; 395:912-920.

4. Matson JL, Minshawi NF. Early intervention for autism spectrum disorders: A critical analysis: Elsevier, 2006.

5. DeFilippis M, Wagner KD. Treatment of Autism Spectrum Disorder in Children and Adolescents. Psychopharmacol Bull 2016; 46:18-41.

6. Beavers GA, Iwata BA, Lerman DC. Thirty years of research on the functional analysis of problem behavior. J Appl Behav Anal 2013; 46:1-21.

7. Steinman G. COVID-19 and autism. Med Hypotheses 2020; 142:109797.

8. Amaral DG, de Vries PJ. COVID-19 and autism research: perspectives from around the globe. Autism Res 2020; 13:844869.

9. Rodriguez KA. Maintaining Treatment Integrity in the Face of Crisis: A Treatment Selection Model for Transitioning Direct ABA Services to Telehealth. Behav Anal Pract 2020; 13:1-8. 